\title{
Atrial Deformation Correlated with Functional Capacity in Mitral Stenosis Patients
}

\author{
Monir Osman Amin ${ }^{1}$, Mohamed Saad Al Gammal ${ }^{1}$, \\ Mohamed Said Ewais Mohamed ${ }^{2, *}$ \\ ${ }^{1}$ Cardiology Department, Al-Azhar University, Cairo, Egypt \\ ${ }^{2}$ Cardiology Department of Mabaret misr Alkadema Hospital, Cairo, Egypt \\ Email address: \\ mselgamal@yahoo.com (M. S.Al Gammal),drm7mds3id@yahoo.com (M. S. E. Mohamed) \\ ${ }^{*}$ Corresponding author
}

\section{To cite this article:}

Monir Osman Amin, Mohamed Saad Al Gammal, Mohamed Said Ewais Mohamed. Atrial Deformation Correlated with Functional Capacity in Mitral Stenosis Patients. Cardiology and Cardiovascular Research. Vol. 4, No. 3, 2020, pp. 152-163. doi: 10.11648/j.ccr.20200403.21

Received: July 8, 2020; Accepted: August 3, 2020; Published: August 25, 2020

\begin{abstract}
Background: Mitral stenosis (MS) is the most common valve disease seen as a sequel of rheumatic fever and usually presents with exertional dyspnea, right sided heart failure and pulmonary arterial hypertension. Aim: To determine the functional class of dyspnea in patients with MS and to investigate the relationship between Left Atrial (LA) deformation as measured by two-dimensional Speckle Tracking Echocardiography (STE)-derived LA strain and heart failure symptoms. Patients and methods: This was a prospective study carried out on 25 patients referred for evaluation of MS using echocardiography and 25 control subjects, who were evaluated using 2D and color Doppler echocardiography, including STE-derived LA strain (LAS) and peak positive filling strain rate (LASRr) during the LA reservoir phase. Those results were then correlated with New York Heart Association (NYHA) functional class. Results: There was a statistically significant difference between the patients and the control groups in the LAS in the apical 4 chamber view (LAS A4) (64.240 \pm 15.271 , $40.360 \pm 21.785$, respectively; $p=0.001)$, LASRr A4 (2.504 $\pm 0.358,1.740 \pm 0.716$, respectively; $p=0.001)$ and LASRc A4 $(-2.796 \pm 0.226,-1.820 \pm 0.928$, respectively; $\mathrm{p}=0.001)$. also, there was a highly significant relation between NYHA class and LAS A4, LASRr A4 and Peak Negative Conduit Strain Rate (LASRc A4), with $\mathrm{P}<0.001$ with all data. Conclusion: This study showed that all left atrial (LA) reservoir, conduit and contractile functions showed prominent impairment in mitral stenosis patients. Speckle tracking strain and strain rate were useful in pointing out the different effects of MS on left atrial functions. The assessment of LA dimension and function can provide further insights in patients with MS, LA dimension and volume in patients with MS had a positive significant correlation with NYHA classes (heart failure symptoms). In stable patients (NYHA classes I \& II) with MS, NYHA functional class independently correlated with LAS and LASRr as LAS and LASRr values of stable patients and control aspects were normal. While LA deformation (LAS and LASRr) was significantly correlated with heart failure symptoms (NYHA Classes III \& IV) in patients with MS.
\end{abstract}

Keywords: Mitral Stenosis, Left Atrium, Speckle Tracking Echocardiography

\section{Introduction}

The obstruction of antegrade flow from the left atrium (LA) to the left ventricle results in pressure and volume overload of the LA. Mitral stenosis (MS) severity can be graded by Echocardiography; however, heart failure symptoms do not correlate with echocardiographic parameters [1].

Proper atrial function plays an important role in determining atrial pressure and in maintaining blood flow across the stenotic valve. Previous studies have demonstrated that left atrium (LA) reservoir function assessed by tissue Doppler strain rate imaging had prognostic value in asymptomatic patients with rheumatic MS [1]. The newly developed speckle tracking echocardiography (STE) is useful in assessing LA strain and strain rate [2]. A previous study demonstrated good correlation between tissue Doppler-derived LA strain and STE-derived LA strain, but found less intra- and inter-observer variability using STE [3].

As the target organ affected by MS, LA remodeling occurs early. Increased LA pressure within the LA myocardium may lead to progressive intrinsic LA dysfunction. But in the early 
stage, the initial rise in LA contribution to LV filling may represent a compensatory response to the diminution of the rapid early component of LV filling, resulting in an elevated left atrial filling component as assessed by conventional Doppler echocardiography [4]. It is difficult to detect LA dysfunction by traditional echocardiography. Noninvasive quantification of LA function using SR imaging enables the evaluation of LA dysfunction even at this stage [5].

The stenotic mitral valve leaflet causes an abnormal pressure gradient between the LA and the LV. The elevated LA pressure raises the pulmonary venous and capillary pressure, resulting in exertional dyspnea. However, different LA pressures were noted despite similar MV areas because LA compliance is also an important determinant of LA pressure [6]. Both atrial stiffness and atrial reservoir dysfunction were affected by varying degrees of atrial muscle bundle disorganization and atrial fibrosis [7].

LA dysfunction (represented as impaired LA deformation using two-dimensional [2D] strain imaging) has been observed in association with rheumatic MS. However, the clinical role of STE-derived LA strain has rarely been studied in MS [8].

This study aimed to determine the functional class of dyspnea in patients with mitral stenosis and to investigate the relationship between left atrial deformation as measured by two-dimensional STE-derived LA strain and heart failure

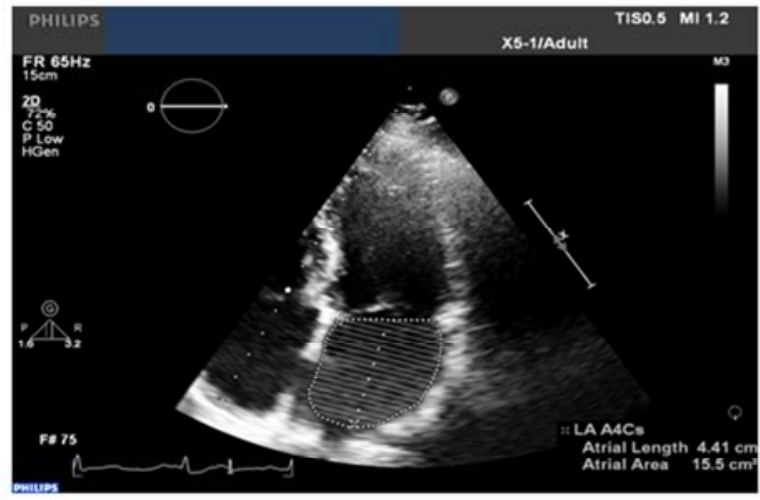

(a) symptoms.

\section{Patients and Methods}

This study had been conducted in Mabaret Misr Alkadema Hospital from January 2019 to October 2019. All subjects gave their verbal consent for the participation in the study.

This prospective case-control study included 50 subjects, who were categorized into two groups: Group 1: Included 25 patients with history of Mitral valve stenosis, from the cardiology outpatient clinic of the hospital. Group 2: Included 25 healthy individuals recruited as control group.

The control group is defined as any individual with no cardiovascular disease or risk factors, who had normal cardiovascular examination and transthoracic echocardiography.

\subsection{Inclusion Criteria}

Mitral valve stenosis patients of both sexes and above 10 years.

\subsection{Exclusion Criteria}

Inadequate or incomplete echocardiography images. Patients with congenital heart disease and patients with previous history of cardiac surgery.

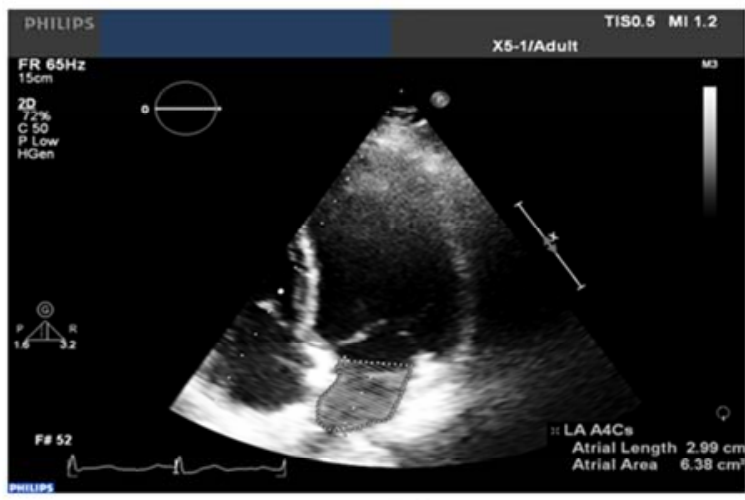

(b)

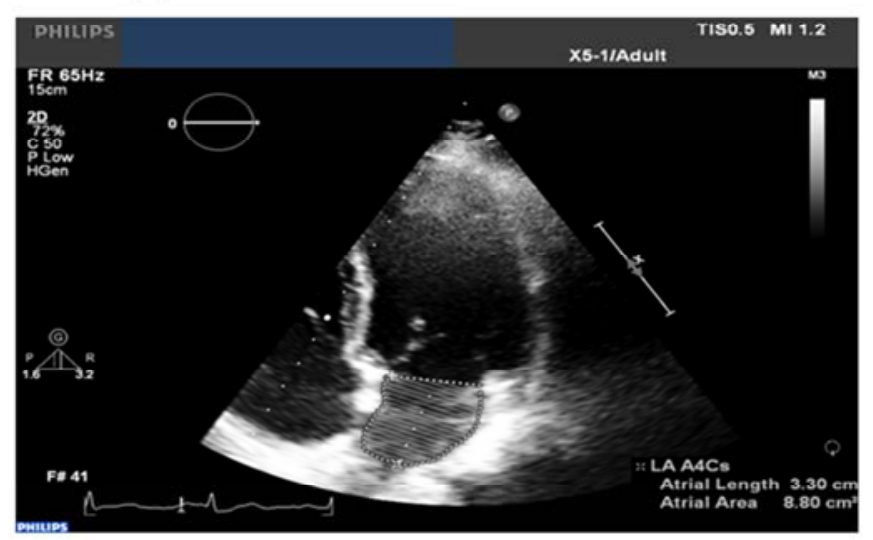

(c)

Figure 1. a) Maximal LA volume, b) minimal LA volume and c) preatrial contraction volume for a patient in the apical 4 chamber view (from our study). 


\subsection{All Patients Were Subjected to the Following}

Full medical history, including: Age, gender. symptoms suggestive of complications of mitral valve disease according to NYHA Functional Classification; Full clinical examination; 12 Leads surface electrocardiogram (ECG) and Echocardiographic examination: All patients have been evaluated by transthoracic echocardiography, including conventional, color, Pulsed and Continuous Wave Doppler, Planimetry and Tissue Doppler Imaging (TDI), and Strain and Strain rate analysis by 2D Speckle tracking of the LA:

Conventional Echocardiographic measurements: Left ventricle dimensions, LV ejection fraction (EF), LA Dimension (LAD) and LA volumes using biplane modified Simpson's method of discs. (Figure 1)
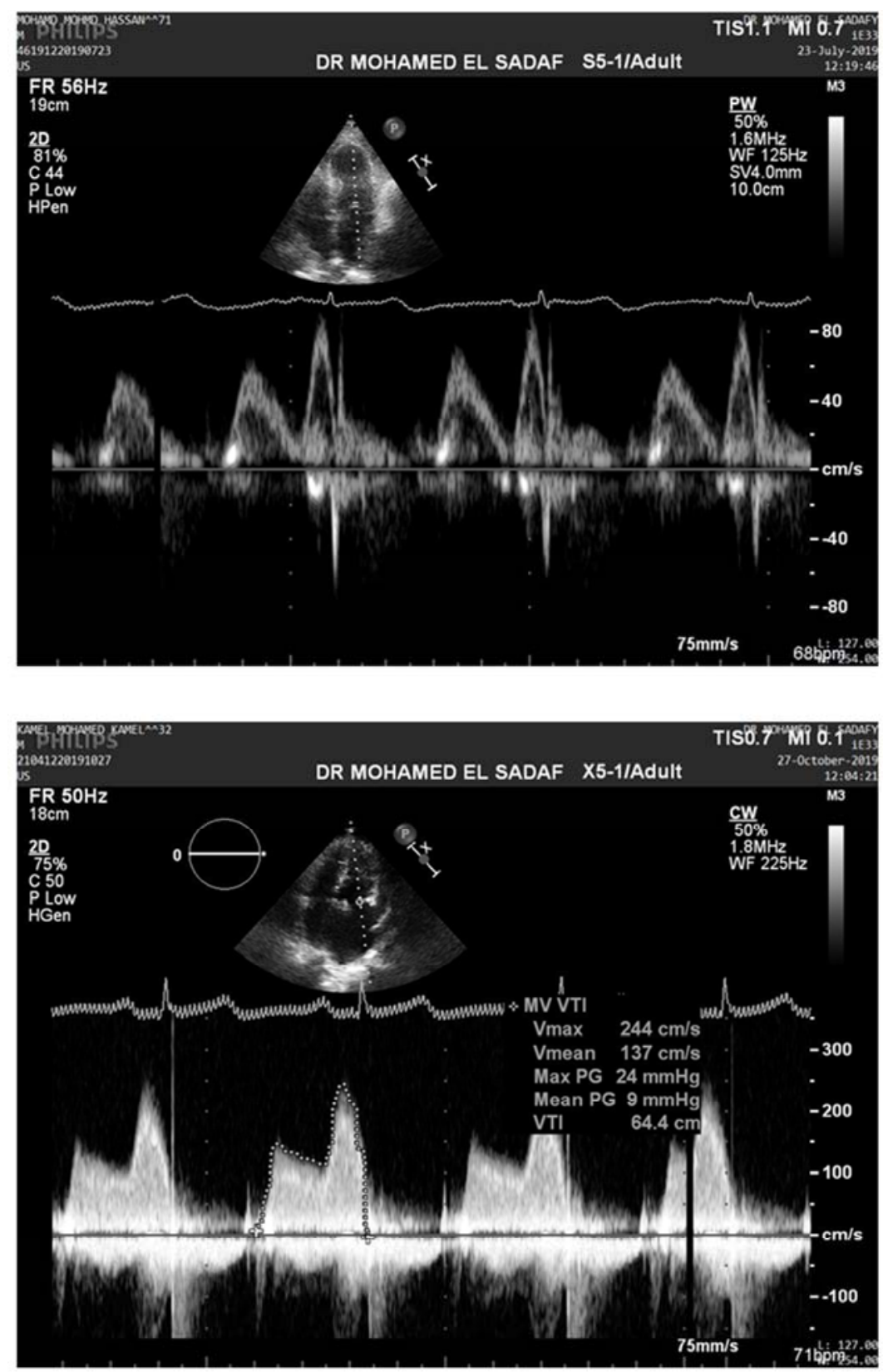

Figure 2. Pulsed and Continuous Wave Doppler methods to quantify mitral stenosis (from our study).

Pulsed and Continuous Wave Doppler: Transmitral inflow velocities (Figures 2\&3): Pulsed Wave (PW) Doppler can localize the velocity at a certain point, but it is subjected to the phenomena of aliasing, so it cannot quantify the high velocity jets. While Continuous Wave (CW) Doppler cannot localize the velocity at a certain point, but it can record the very high velocity jets. So, CW Doppler was used to quantify the severity of MS by measuring the Mean Pressure Gradient (MPG) and Pressure Half Time (PHT) method. Also, Mitral stenosis was quantified by planimetry (Figure 4) and Estimated Pulmonary Artery Systolic Pressure (EPASP). The mitral valve area was calculated by one or more of these 
methods and was considered as the classification in Table 1.

Table 1. Classification of the severity of mitral stenosis. [9]

\begin{tabular}{llll}
\hline & Mild & Moderate & Severe \\
\hline Mean gradient $(\mathrm{mmHg})$ & Less than 5 & $5-10$ & Greater than 10 \\
Pulmonary artery systolic pressure $(\mathrm{mmHg})$ & Less than 30 & $30-50$ & Greater than 50 \\
Valve area $\left(\mathrm{cm}^{2}\right)$ & Greater than 1.5 & $1.0-1.5$ & Less than 1.0 \\
\hline
\end{tabular}

Tissue Doppler Imaging (TDI) derived mitral annular velocities: Mitral annular velocities were evaluated by placing the sample volume at the lateral and septal mitral annulus in the apical 4-chamber view (A4). Peak velocities of the early $\left(E^{\prime}\right)$ and late $\left(A^{\prime}\right)$ diastolic phases, and systolic (S) wave of the septal and lateral mitral annulus were measured. The E/E' ratio was calculated in Figure 5.

Two Dimensional Speckle Tracking Strain and Strain Rate Analysis: For speckle tracking analysis, apical four and two-chamber views were recorded. For each view, adequate gray scale images were obtained to outline the endocardial border of the LA. (Figures $6 \& 7$ )

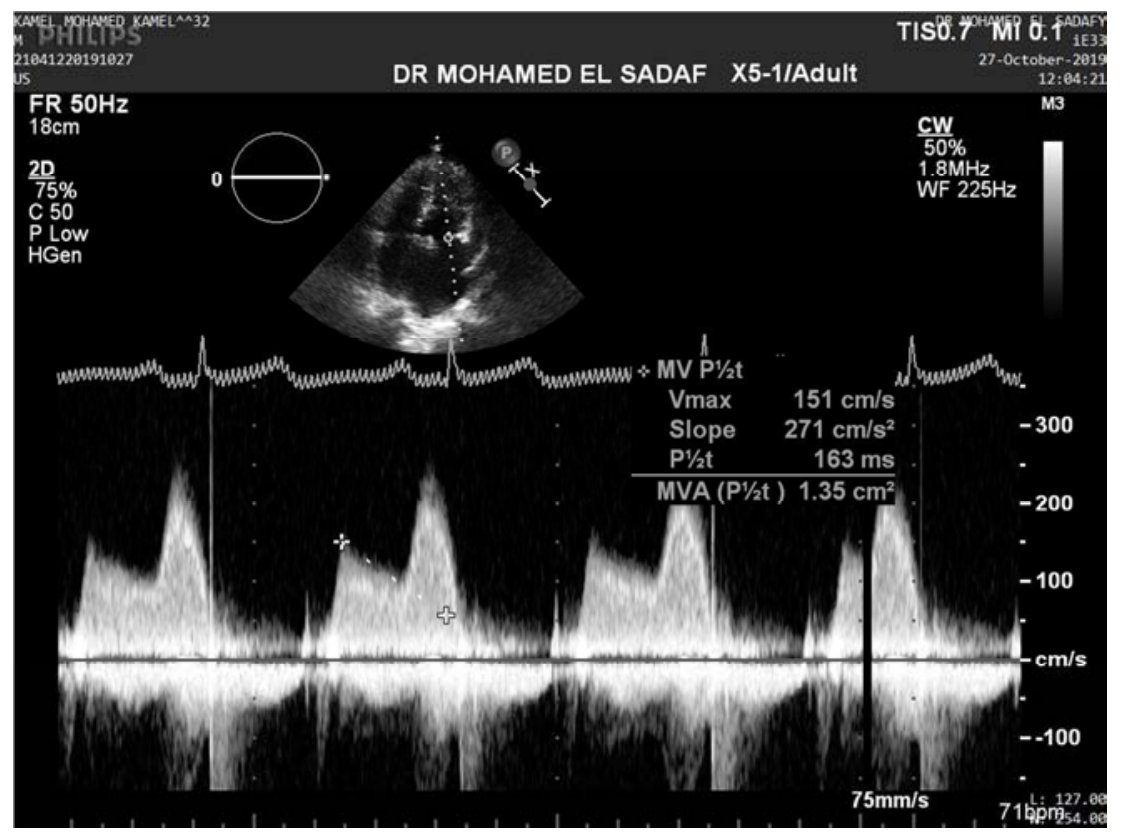

Figure 3. Calculation of Mitral Valve Area (MVA) by Pressure Half Time (PHT) method (from our study).

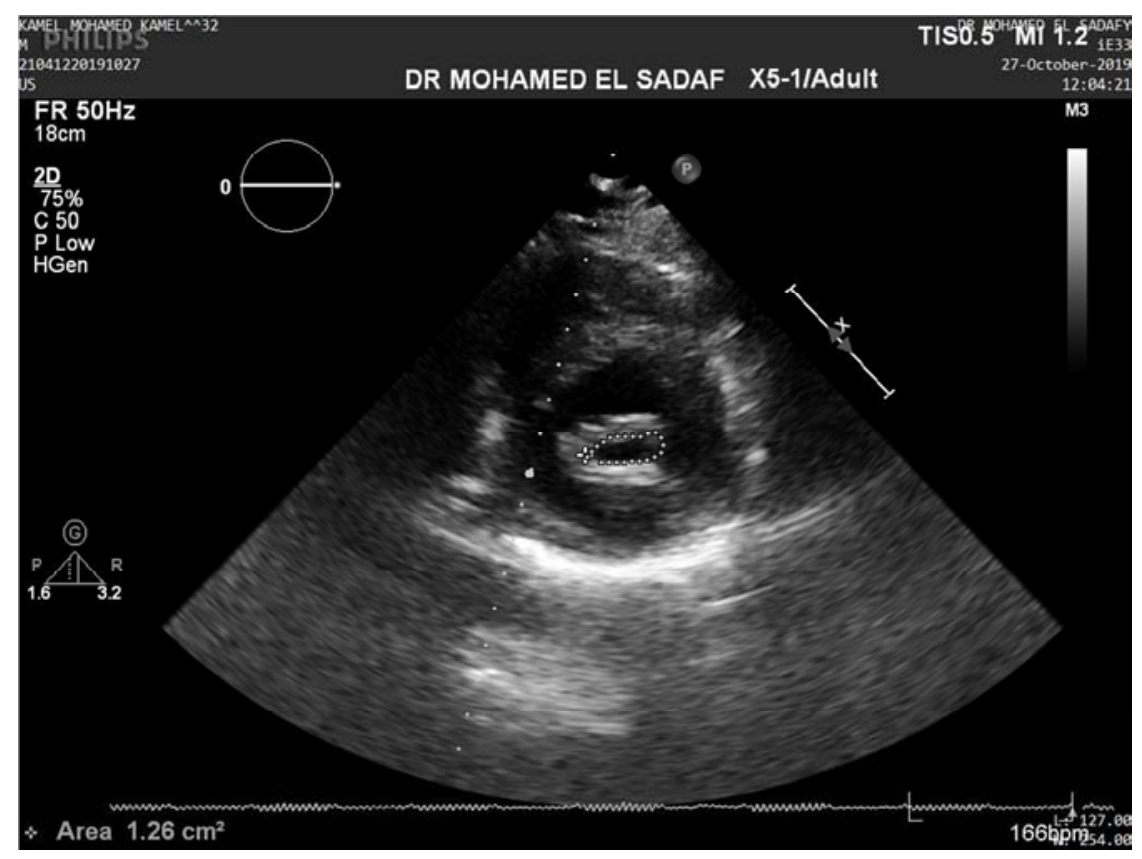

Figure 4. Tracing of Mitral Valve Area (MVA) by Planimetry method in Parasternal Short Axis (PSAX) view (from our study). 
The following parameters were measured: we prospectively measured LA diameter and volume, mitral valve area, LVEF, diastolic dysfunction, transmitral MPG and EPASP. Using STE, we measured the left atrial four-chamber (A4) global strain, LASRr, LASRc, and the left atrial two-chamber (A2) global strain, LASRr, LASRc.

Statistical analysis: Results of the present study were statistically analyzed using SPSS 25 (IBM, USA). Data were represented as median (interquartile range) or number and percentage. Numerical data were compared using Mann-Whitney U test, while categorical data were compared using Fisher's exact test or Chi-square test, as appropriate. $\mathrm{P}$ value less than 0.05 was considered statistically significant.

\section{Results}

Table 2. Comparison between two groups according to the gender.

\begin{tabular}{|c|c|c|c|c|c|}
\hline & & & \multicolumn{2}{|l|}{ Groups } & \multirow{2}{*}{ Total } \\
\hline & & & Control & Patients & \\
\hline \multirow{4}{*}{ Sex } & \multirow{2}{*}{ Female } & $\mathrm{N}$ & 14 & 12 & 26 \\
\hline & & $\%$ & $56.0 \%$ & $48.0 \%$ & $52.0 \%$ \\
\hline & \multirow{2}{*}{ Male } & $\mathrm{N}$ & 11 & 13 & 24 \\
\hline & & $\%$ & $44.0 \%$ & $52.0 \%$ & $48.0 \%$ \\
\hline \multirow{2}{*}{ Total } & & $\mathrm{N}$ & 25 & 25 & 50 \\
\hline & & $\%$ & $100.0 \%$ & $100.0 \%$ & $100.0 \%$ \\
\hline \multirow{2}{*}{ Chi-square } & & $X^{2}$ & 0.321 & & \\
\hline & & $\mathrm{P}$-value & 0.571 & & \\
\hline
\end{tabular}

As demonstrated in Table 2, the gender of subjects in control group shows that more than half of subjects investigated were female $14(56 \%)$, while the gender of patients in patients group shows that more than half of our patients were male 13 (52\%), with no statistically significant differences between the two groups $(\mathrm{P}=0.571)$.

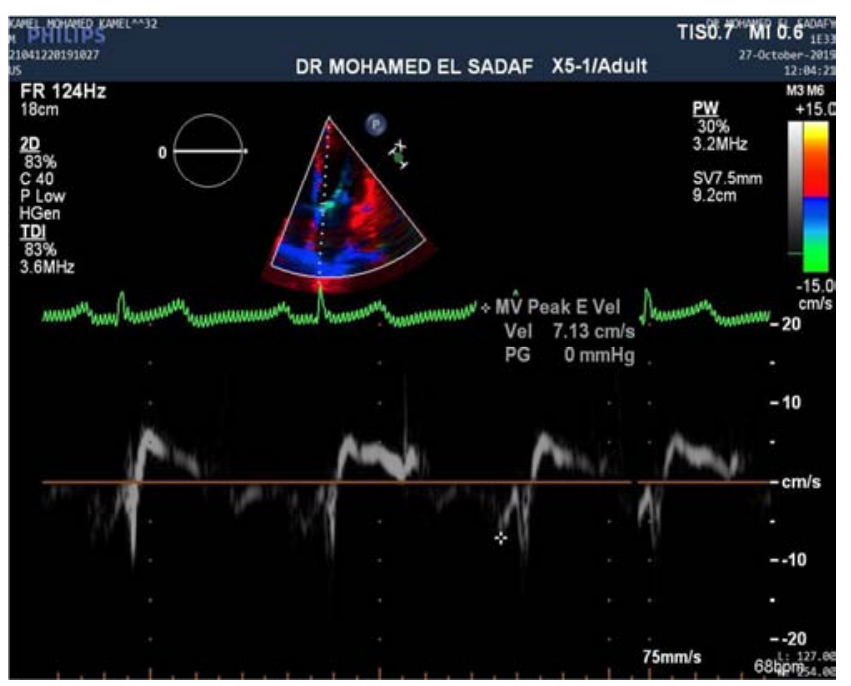

Figure 5. Tissue Doppler imaging of septal mitral annulus in mitral stenosis patient. (from our study).

Table 3. Correlation between the different data.

\begin{tabular}{|c|c|c|c|c|c|c|c|}
\hline Correlation & & LAS A4 & LASRr A4 & LASRc A4 & LAS A2 & LASRr A2 & LASRc A2 \\
\hline \multirow{2}{*}{ LV EF } & $\mathrm{R}$ & 0.124 & 0.019 & -0.113 & 0.078 & -0.029 & 0.091 \\
\hline & P-value & 0.555 & 0.927 & 0.591 & 0.711 & 0.891 & 0.665 \\
\hline \multirow{2}{*}{ LA Volume } & $\mathrm{R}$ & -0.841 & -0.845 & 0.874 & -0.768 & -0.720 & 0.796 \\
\hline & P-value & $<0.001^{*}$ & $<0.001 *$ & $<0.001 *$ & $<0.001^{*}$ & $<0.001^{*}$ & $<0.001 *$ \\
\hline \multirow{2}{*}{ PASP } & $\mathrm{R}$ & -0.560 & -0.439 & 0.457 & -0.586 & -0.465 & 0.478 \\
\hline & P-value & $0.004 *$ & $0.028 *$ & $0.022 *$ & $0.002 *$ & $0.019 *$ & $0.016^{*}$ \\
\hline MPG & $\mathrm{R}$ & -0.614 & -0.566 & 0.582 & -0.676 & -0.483 & 0.499 \\
\hline \multirow{2}{*}{ LAD } & $\mathrm{R}$ & -0.544 & -0.519 & 0.510 & -0.648 & -0.672 & 0.723 \\
\hline & P-value & $0.005^{*}$ & $0.008 *$ & $0.009 *$ & $<0.001^{*}$ & $<0.001 *$ & $<0.001 *$ \\
\hline \multirow{2}{*}{ MVA } & $\mathrm{R}$ & 0.545 & 0.579 & -0.677 & 0.590 & 0.538 & -0.633 \\
\hline & P-value & $0.005^{*}$ & $0.002 *$ & $<0.001 *$ & $0.002 *$ & $0.005^{*}$ & $<0.001 *$ \\
\hline \multirow{2}{*}{ Age } & $\mathrm{R}$ & -0.046 & 0.091 & -0.161 & -0.058 & 0.099 & -0.118 \\
\hline & $\mathrm{P}$-value & 0.826 & 0.665 & 0.442 & 0.784 & 0.637 & 0.575 \\
\hline
\end{tabular}

[*] statistically significant at $\mathrm{P}$-value $\leq 0.05$.

Table 3 shows a correlation between the different data, and it was found that LAS A4, LAS A2, LASRr A4 and LASRr A2 had a highly significant negative correlation with LA volume, PASP, MPG and LAD. However, LAS A4, LAS A2, LASRr A4 and LASRr A2 had a highly significant positive correlation with MVA. LASRc A4 and LASRc A2 had a highly significant negative correlation with MVA while both had a highly significant positive correlation with LA volume, PASP, MPG and LAD.

Table 4. Correlation between the different data (Figures 8\&9).

\begin{tabular}{|c|c|c|c|c|c|c|}
\hline Correlation & & LAS A4 & LASRr A4 & LASRc A4 & LAS A2 & LASRr A2 \\
\hline \multirow{2}{*}{ LASRr A4 } & $\mathrm{R}$ & 0.891 & & & & \\
\hline & P-value & $<0.001^{*}$ & & & & \\
\hline \multirow{2}{*}{ LASRc A4 } & $\mathrm{R}$ & -0.904 & -0.914 & & & \\
\hline & $\mathrm{P}$-value & $<0.001 *$ & $<0.001^{*}$ & & & \\
\hline \multirow{2}{*}{ LAS A2 } & $\mathrm{R}$ & 0.890 & 0.832 & -0.845 & & \\
\hline & P-value & $<0.001 *$ & $<0.001 *$ & $<0.001^{*}$ & & \\
\hline LASRr A2 & $\mathrm{R}$ & 0.834 & 0.904 & -0.834 & 0.864 & \\
\hline
\end{tabular}




\begin{tabular}{|c|c|c|c|c|c|c|}
\hline Correlation & & LAS A4 & LASRr A4 & LASRc A4 & LAS A2 & LASRr A2 \\
\hline \multirow{3}{*}{ LASRc A2 } & P-value & $<0.001 *$ & $<0.001 *$ & $<0.001 *$ & $<0.001 *$ & \\
\hline & & -0.830 & -0.865 & 0.890 & -0.879 & -0.907 \\
\hline & P-value & $<0.001 *$ & $<0.001 *$ & $<0.001 *$ & $<0.001 *$ & $<0.001^{*}$ \\
\hline
\end{tabular}

[*] statistically significant at $\mathrm{P}$-value $\leq 0.05$.

Table 4 shows correlation between the different data, and it was found that LAS A4 and LASRr A4 had a highly significant negative correlation with each of LASRc A4 and LASRc A2. LASRc A4 had a highly significant negative correlation with each of LAS A2 and LASRr A2. There is a highly significant negative correlation between LASRc A2 and each of LAS A2 and LASRr A2. LAS A4 had a highly significant positive correlation with LASRr A4, LAS A2 and LASRr A4. LASRr A4 had a highly significant positive correlation with each of LAS A2 and LASRr A2. LASRc A4 had a highly significant positive correlation with LASRc A2. LAS A2 had a highly significant positive correlation with LASRr A2.

Table 5. Relation between NYHA classes and different data.

\begin{tabular}{|c|c|c|c|c|c|c|c|c|c|c|c|c|c|c|}
\hline & \multicolumn{12}{|l|}{ NYHA } & \multirow{2}{*}{\multicolumn{2}{|c|}{ ANOVA }} \\
\hline & \multicolumn{3}{|l|}{ I } & \multicolumn{3}{|l|}{ II } & \multicolumn{3}{|l|}{ III } & \multicolumn{3}{|l|}{ IV } & & \\
\hline & Mean & \pm & SD & Mean & \pm & SD & Mean & \pm & SD & Mean & \pm & SD & $\mathbf{F}$ & P-value \\
\hline LAS A4 & 66.714 & \pm & 14.482 & 51.684 & \pm & 17.932 & 27.286 & \pm & 12.148 & 13.667 & \pm & 3.055 & 18.631 & $<0.001^{*}$ \\
\hline MVA & 4.271 & \pm & 0.816 & 2.344 & \pm & 1.266 & 1.333 & \pm & 0.576 & 0.833 & \pm & 0.153 & 26.135 & $<0.001^{*}$ \\
\hline LAD & 3.368 & \pm & 0.327 & 4.137 & \pm & 0.361 & 4.583 & \pm & 0.755 & 5.000 & \pm & 0.300 & 26.305 & $<0.001^{*}$ \\
\hline MPG & 0.571 & \pm & 0.926 & 3.789 & \pm & 2.594 & 8.000 & \pm & 1.000 & 10.333 & \pm & 3.055 & 43.583 & $<0.001^{*}$ \\
\hline PASP & 17.095 & \pm & 4.369 & 29.211 & \pm & 6.663 & 37.571 & \pm & 3.309 & 44.333 & \pm & 1.155 & 46.695 & $<0.001^{*}$ \\
\hline LV EF & 61.810 & \pm & 5.879 & 56.789 & \pm & 8.162 & 54.429 & \pm & 9.163 & 42.000 & \pm & 5.292 & 7.415 & $<0.001^{*}$ \\
\hline LASRr A4 & 2.552 & \pm & 0.360 & 2.058 & \pm & 0.531 & 1.600 & \pm & 0.737 & 0.733 & \pm & 0.208 & 16.246 & $<0.001^{*}$ \\
\hline LASRc A4 & -2.852 & \pm & 0.087 & -2.274 & \pm & 0.717 & -1.543 & \pm & 0.842 & -0.500 & \pm & 0.000 & 22.608 & $<0.001^{*}$ \\
\hline LAS A2 & 67.619 & \pm & 12.428 & 48.579 & \pm & 13.906 & 30.857 & \pm & 14.747 & 12.333 & \pm & 2.517 & 25.652 & $<0.001^{*}$ \\
\hline LASRr A2 & 2.586 & \pm & 0.362 & 2.016 & \pm & 0.478 & 1.671 & \pm & 0.772 & 0.833 & \pm & 0.058 & 16.540 & $<0.001^{*}$ \\
\hline LASRc A2 & -2.857 & \pm & 0.081 & -2.426 & \pm & 0.707 & -1.671 & \pm & 1.112 & -0.767 & \pm & 0.289 & 14.793 & $<0.001^{*}$ \\
\hline
\end{tabular}

[*] statistically significant at $\mathrm{P}$-value $\leq 0.05$.

Table 5 shows the relation between NYHA class and different data, and there was a highly significant negative correlation between NYHA class and each of LAS A4, MVA, LV EF, LASRr A4, LASRc A4, LAS A2, LASRr A2 and LASRc A2 with $(\mathrm{P}<0.001)$ with all data; while there was a highly significant positive correlation between NYHA class and each of LAD, MPG, PASP and LA volume with $(\mathrm{P}<0.001)$ with all data.

\section{Discussion}

This was a prospective study carried out on 25 patients referred for evaluation of MS using echocardiography matched with 25 control subjects. As regard to demographic characteristics, more than half of the subjects in the control group were females $14(56 \%)$, while in the patients group, more than half of patients were males 13 (52\%) with no statistically significant differences between the two groups, where $\mathrm{P}=0.571$. The age in the control group was ranged between 17-67 years old with a mean value of $(37.08 \pm 13.366)$, while in the patients group, it was ranged between 20-71 years old with a mean value of (43.08 \pm 13.678$)$, with no statistically significant differences between the two groups where $\mathrm{P}=0.123$.

Comparing to the study of Mohammed et al [10] that was conducted on 44 patients having systolic HF, where mean age was $55.7 \pm 8.6$ years, $79 \%$ of them were males.

As regard to NYHA classification, subjects in the control group showed that most of patients investigated $20(80 \%)$ had NYHA class I and only 5 (20\%) had NYHA class II, while in the patients group, more than half of patients investigated $14(56 \%)$ had NYHA class II, 7 (28\%) had NYHA class III, 3 (12\%) had NYHA class IV and only one patients had NYHA class I with statistically significant differences between the two groups, where $\mathrm{P}=0.0001$.

In another study conducted by Mohammed et al [10] where study subjects were classified according to their NYHA functional class into two groups (group I related to NYHA class II, and group II related to NYHA class III/ambulatory class IV). The average NYHA class for the whole patients was $2.75 \pm 0.7$, with a median of 3 and inter quartile range $(\mathrm{IQR})=1$. Both groups were age and sex matched.

Reda et al [11] reported that there was a negative correlation between all LA parameters measured by strain (peak atrial longitudinal strain (PALS), peak atrial contraction strain (PACS), postatrial contraction (Post-A), and LA contraction systolic index (LA CSI) in septal, lateral, anterior, and inferior walls, and NYHA class. A statistically significant correlation $(P<0.05)$ was found between PALS of LA lateral, anterior, inferior walls, and NYHA. Also they found a significant negative correlation between PALS of the lateral atrial wall and NYHA class.

Which is concordant with the current study, which demonstrated that LAS A4, LAS A2, LASRr A4 and LASRr A2 had a highly significant negative correlation with LA volume, PASP, MPG and LAD. However, LAS A4, LAS A2, LASRr A4 and LASRr A2 had a highly significant positive 
correlation with MVA. LASRc A4 and LASRc A2 had a highly significant negative correlation with MVA, while both had a highly significant positive correlation with LA volume, PASP, MPG and LAD.

Also, in our study, we assessed the relation between NYHA class and different data, and there was a highly significant negative correlation between NYHA class and each of LAS A4, MVA, LV EF, LASRr A4, LASRc A4, LAS A2, LASRr A2 and LASRc A2 with $(\mathrm{P}<0.001)$ with all data; while there was a highly significant positive correlation between NYHA class and each of LAD, MPG, PASP and LA volume with $(\mathrm{P}<0.001)$ with all data.

Which is concordant with Leung DY et al [12], where the enlargement of the LA and the increase in the LA emptying fraction are adaptive responses to the impaired LV diastolic function to maintain normal LV filling pressures. Decreased LA compliance with reduced reservoir and contractile pump functions can counteract this adaptive mechanism and promote the occurrence of symptoms.
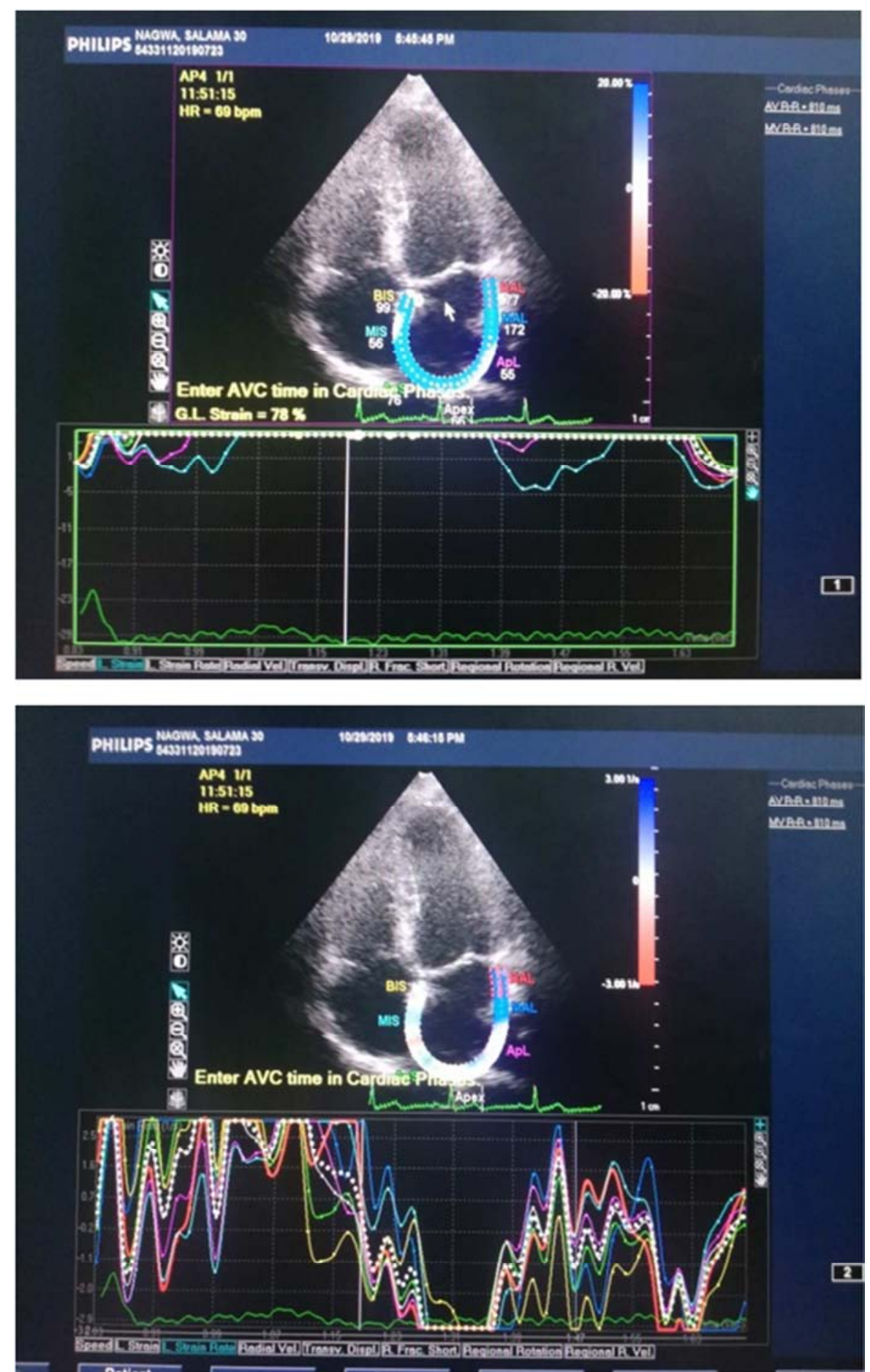

Figure 6. Strain and strain rate in apical 4 chamber view in normal subject. (from our study). 

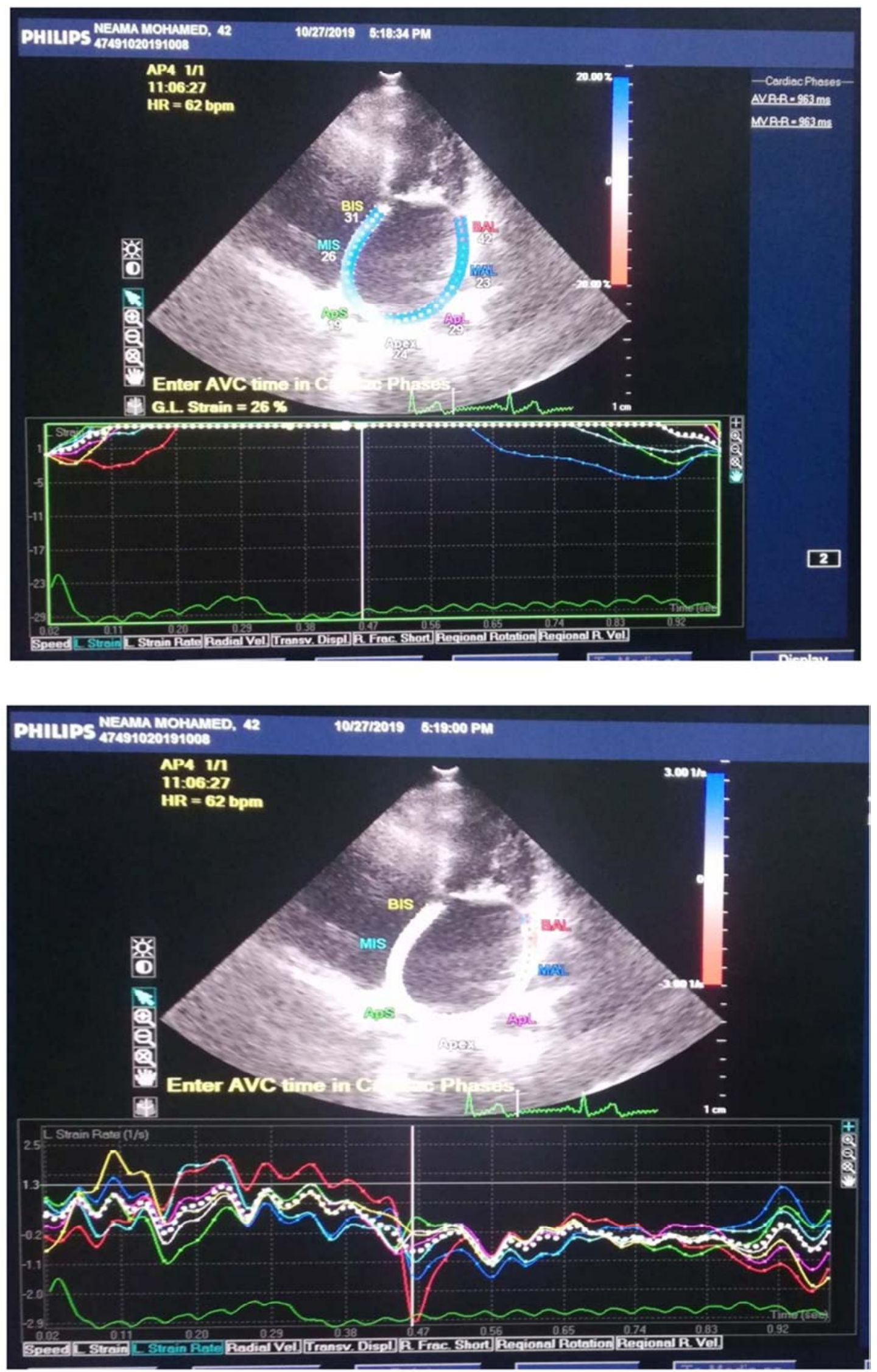

Figure 7. Strain and strain rate in apical 4 chamber view in mitral stenosis patient. (from our study). 

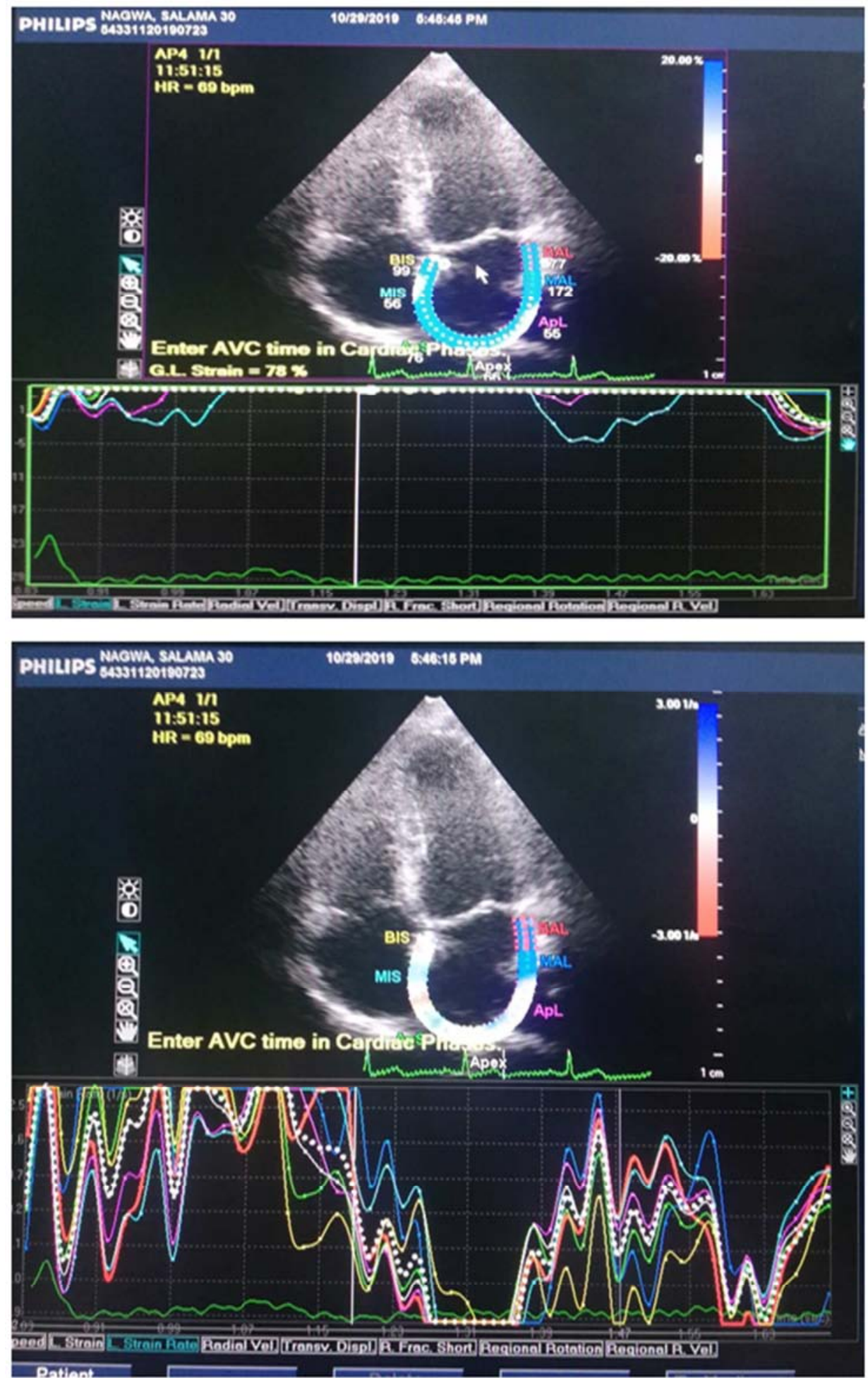

Figure 8. The figure shows strain LAS (upper panel) and strain rate LASRr and LASRc (lower panel) in the apical 4 chamber view (A4) by using speckle tracking echocardiography of control subject. (from our study).

Also, in concordance with Mohammed et al [10] that reported that there were negative correlations between the NYHA class and PALS $(r=-0.436, P=0.003)$ PACS $(r=-0.48$, $P=0.001)$, and $\mathrm{LV}$ global longitudinal strain $(r=-0.30, P=0.05)$. There was a positive correlation between PALS and the average LV annular peak systolic velocity $\left(S^{\prime}\right) \quad(r=0.35$, $P=0.019)$ and LA late peak atrial diastolic velocity (LA $\left.a^{\prime}\right)$
( $r=0.367, P=0.014)$.

Numerous studies had confirmed the strong association between LA function and the functional capacity. 2D-STE of LA strain had shown to be an independent predictor of exercise capacity in both heart failure with reduced ejection fraction $(\mathrm{HFrEF})$ and heart failure with preserved ejection fraction (HFpEF) Kusunose et al [13]. While, D'Andrea et al 
[14] demonstrated that PALS was an independent predictor of estimated metabolic equivalents in patients with $\mathrm{HFpEF}$ and HFrEF.

Similar result was reported by Ahmed et al [15], where a significant decrease in LA strain parameter of LA function assessed by 2D-STE in systolic HF, and the study demonstrated that LA reservoir and booster pump function correlate negatively with heart failure symptoms and positively with LV global strain.
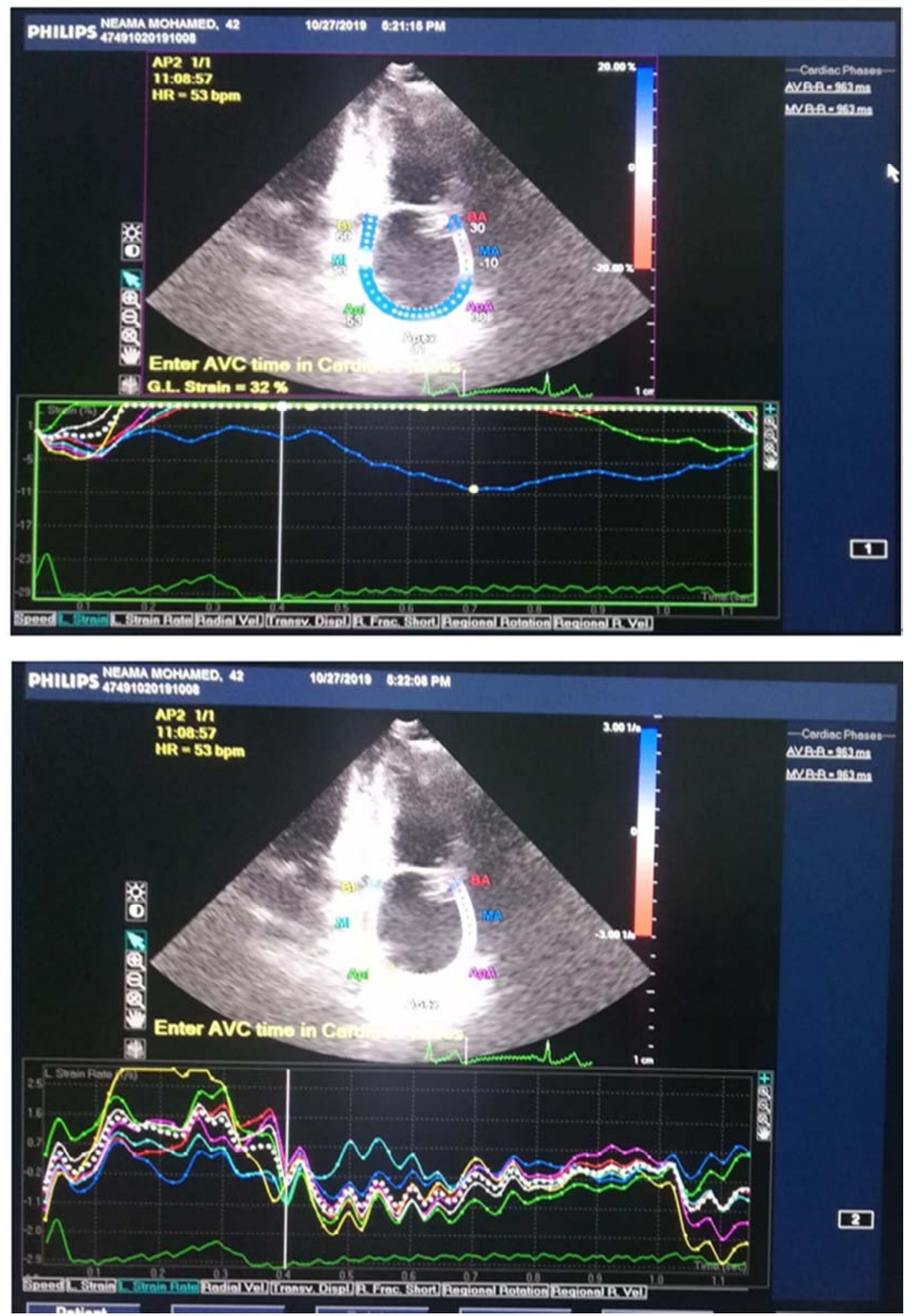

Figure 9. The figure shows strain LAS (upper panel) and strain rate LASRr and LASRc (lower panel) in the apical 2 chamber view (A2) by using speckle tracking echocardiography of mitral stenosis patient. (from our study). 
The current study revealed that, in the control group, LAD was ranged between $(2.7-4.5 \mathrm{~cm})$ with a mean value of (3.473 \pm 0.406$)$, while in the patients group, it was ranged between $(3-5.30 \mathrm{~cm})$ with a mean value of $(4.383 \pm 0.564)$ with a statistically significant differences between the control and the patients groups $(\mathrm{P}<0.001)$.

This is concordant with the study by Rahimtoola SH et al [16] where in MS patients, LAD increases with the increment of pressure gradient between the left atrium and left ventricle; and the chance of left atrial mural thrombi increases with the increment of left atrial size. Two-dimensional echocardiography is a well-established technique to assess the left atrial size. LAD is measured by M-mode echocardiography in the standard parasternal long axis view and enlarged LAD in rheumatic mitral stenosis is seen in echocardiography, Left atrial size enlarges in MS and previously it was reported that the left atrial size enlargement is an independent risk factor for the left atrial thrombi and it is an indication for anticoagulation therapy for MS patients.

The current study showed that LAD and volume had a positive significant correlation with NYHA class with $\mathrm{P}<0.001$, and LAD and volume showed a significant correlation between the two groups with $\mathrm{P}<0.001$. Which is concordant with the study by Bianca Moise et al [17], where MS leads to extensive LA remodeling, having important structural and functional consequences with implications over LA mechanical, electrical (the occurrence of atrial fibrillation) and Neurohormonal function. the assessment of LA dimension and function can therefore provide further insights in patients with mitral stenosis, independent of its severity, adding prognostic and clinical value.

Although NYHA classification is to some degree subjective, it is still an easily applied first-line tool in everyday clinical practice to assess patients' functional limitation. Moreover, in more symptomatic patients (NYHA III/IV), the NYHA classification appears to be a well-performing, accurate clinical tool to estimate these patients' functional limitation and guide therapy [10].

\section{Conclusion}

This study showed that all left atrial (LA) reservoir, conduit and contractile functions showed prominent impairment in mitral stenosis patients.

Speckle tracking strain and strain rate were useful in pointing out the different effects of MS on left atrial functions.

The assessment of LA dimension and function can provide further insights in patients with MS, LA dimension and volume in patients with MS had a positive significant correlation with NYHA classes (heart failure symptoms).

In stable patients ( NYHA classes I \& II) with MS, NYHA functional class independently correlated with LAS and LASRr as LAS and LASRr values of stable patients and control aspects were normal. While LA deformation (LAS and LASRr) was significantly correlated with heart failure symptoms (NYHA Classes III \& IV) in patients with MS.

\section{Conflicts of Interest}

All the authors do not have any possible conflicts of interest.

\section{Acknowledgements}

The authors are grateful for the patients without whom this study would not have been done.

\section{References}

[1] Caso P, Ancona R, Di Salvo G, et al. Atrial reservoir function by strain rate imaging in asymptomatic mitral stenosis: prognostic value at 3 year follow-up. Eur $\mathrm{J}$ Echocardiogr. 2009; 10: 753-759.

[2] Saraiva RM, Demirkol S, Buakhamsri A, et al. Left atrial strain measured by two-dimensional speckle tracking represents a new tool to evaluate left atrial function. J Am Soc Echocardiogr. 2010; 23: 172-180.

[3] Tsai WC, Lee CH, Lin CC, et al. Association of left atrial strain and strain rate assessed by speckle tracking echocardiography with paroxysmal atrial fibrillation. Echocardiography. 2009; 26: 1188-1194.

[4] Kono T, Sabbah HN, Rosman H, et al. Left atrial contribution to ventricular filling during the course of evolving heart failure. Circulation. 1992; 86: 1317-1322.

[5] Inaba Y, Yuda S, Kobayashi N, et al. Strain rate imaging for noninvasive functional quantification of the left atrium: comparative studies in controls and patients with atrial fibrillation. J Am Soc Echocardiogr. 2005; 18: 729-736.

[6] Kapoor A, Kumar S, Shukla A, et al. Determinants of left atrial pressure in rheumatic mitral stenosis: role of left atrial compliance and "atrial stiffness". Indian Heart J. 2004; 56: 27-31.

[7] Chien CY, Chen CW, Lin TK, et al. Atrial deformation correlated with functional capacity in mitral stenosis patients. Echocardiography. 2018; 35 (2): 190-195.

[8] Demirkol S, Kucuk U, Baysan O, et al. The impact of mitral stenosis on left atrial function assessed by two-dimensional speckle tracking echocardiography. Echocardiography. 2012; 29: 1064-1070.

[9] Guidelines and standards for Echocardiographic Assessment of Valve Stenosis: EAE/ASE Recommendations for Clinical Practice. Journal of the American Society of Echocardiography January 2009; Vol 22 No 1: 1-23.

[10] Mohammed LA, Agiba NA and Aly AA. Effect of left atrial functions upon the functional capacity in patients with systolic heart failure. Al-Azhar Assiut Med J. 2019; 17: 198-206.

[11] Reda AA, Soliman MA, Ahmed MK, et al. Assessment of left atrial function in patients with systolic heart failure: strain imaging study. Menoufia Med J. 2015; 28: 532-9.

[12] Leung DY, Boyd A, Ng AA, et al. Echocardiographic evaluation of left atrial size and function: current understanding, pathophysiologic correlates, and prognostic implications. Am Heart J. 2008; 15: 1056-1064. 
[13] Kusunose K, Motoki H, Popovic ZB, et al. Independent association of left atrial function with exercise capacity in patients with preserved ejection fraction. Heart. 2012; 98: 1311-1317.

[14] D'Andrea A, Caso P, Romano S, et al. Association between left atrial myocardial function and exercise capacity in patients with either idiopathic or ischemic dilated cardiomyopathy: a two-dimensional speckle strain study. Int J Cardiol. 2009; 132: 354-363.

[15] Ahmed MK, Soliman MA, Reda AA, et al. Assessment of left atrial deformation properties by speckle tracking in patients with systolic heart failure. Egypt Heart J. 2015; 67: 199-208.

[16] Rahimtoola SH, Enriquee-Sarano M, Schaff HV, Frye RL, Mitral valve disease; mitral stenosis. Hurst's The Heart. International edition. McGraw-Hill, Health Professional Division. 1998; Vol. 2, P 1789-1799.

[17] Bianca Moise, Monica Rosca, Dragos Alexandru, Carmen Ginghina, Left atrial remodeling in patients with mitral stenosis. Romanian Journal of Cardiology 2017; Vol. 27, No. 2: 175-176. 